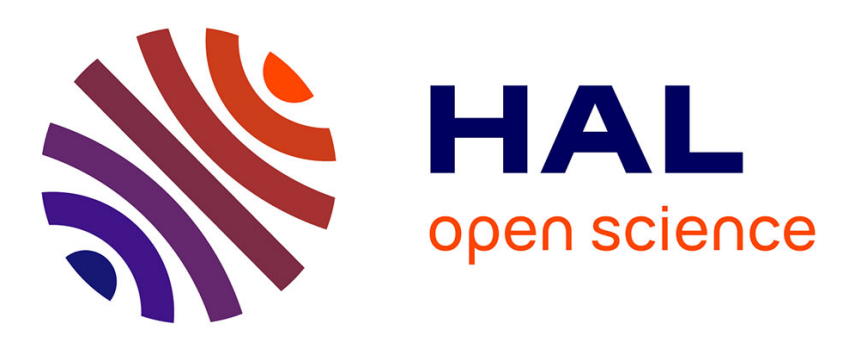

\title{
GTEM cell facility use during project development phases for automotive
}

Olivier Maurice, Frédéric Lafon, Francois de Daran, Rabha Oussedrat, Imad Ben Yacoub

\section{- To cite this version:}

Olivier Maurice, Frédéric Lafon, Francois de Daran, Rabha Oussedrat, Imad Ben Yacoub. GTEM cell facility use during project development phases for automotive. 3rd International Workshop on Electromagnetic Compatibility of Integrated Circuits, Nov 2002, Toulouse, France. pp. 19-22. hal00517749

\author{
HAL Id: hal-00517749 \\ https://hal.science/hal-00517749
}

Submitted on 15 Sep 2010

HAL is a multi-disciplinary open access archive for the deposit and dissemination of scientific research documents, whether they are published or not. The documents may come from teaching and research institutions in France or abroad, or from public or private research centers.
L'archive ouverte pluridisciplinaire HAL, est destinée au dépôt et à la diffusion de documents scientifiques de niveau recherche, publiés ou non, émanant des établissements d'enseignement et de recherche français ou étrangers, des laboratoires publics ou privés. 


\section{GTEM cell facility use during project development phases for automotive Olivier MAURICE-François de DARAN-Frédéric LAFON-Rabha OUSSEDRAT-Imad BEN-YACOUB VALEO Electronic and connective systems - EMC department. 2 rue Fernand Pouillon 94042 Créteil Cedex France}

\begin{abstract}
.
The purpose is to present results done with GTEM facility during the development phases of project, to measure or to quantify emissions and immunity compliance, regarding integrated circuit influence, for automotive standards. These standards use Half Anechoic Chambers (HAC). Studies have already been done with a comparison between TEM cells and HAC or far field emissions, which showed the limitation of TEM facility to correlate with the results in emission with free space field or HAC. We only want to show that the correlation can be good enough to use this facility during a development phase (HAC is often quite overfull and a high price test), how the known of the EMC behavior of the integrated circuit is important to predict the equipment results, and how to use an ICEM model to make prediction regarding emissions.
\end{abstract}

\section{Immunity aspect.}

The first studies done compare the strength during immunity test in GTEM compare to the strength in HAC. The two tests are quite different. Keep in mind that, for a standard automotive test, a 1.5 meter long harness is placed with the equipment under test. In GTEM the field is coupled in propagation mode, from the beginning of the harness to the equipment. Electric and magnetic fields both create current and voltage sources on the wires of the harness. In the HAC standard, electric field coupling is dominant, while magnetic field coupling is taken into account by another test called BCI (Bulk Current Injection). Even with such a redundancy, if an equipment is compliant in the GTEM, it will be compliant in the HAC standard only, without looking for the BCI standard. Until 1 $\mathrm{GHz}$, the energy is principally coupled with the harness. The direct coupled energy between the field and devices on the PCB are very low, because of the low coupling factor of those elements. In this case, it's easy to determine the coupling mechanism between the fields and the harness, whatever is the antenna polarization. The disturbance level of an equipment is determined by the power level induced in differential mode, and conducted to the device input. Now, it has to be confirmed that this level can be the same, or at least higher in GTEM than in HAC.

\section{Test and simulation configuration.}

A first hypothesis on the energy distribution between wires of a harness has to be considered for simulation. Knowing that the current or voltage level can be increased by cross talking phenomenon between two or more wires. We first study a single wire harness to confirm the theoretical models used, and after one wire will be added to complicate the model.

For this test, we rapidly see that if the harness and its product are on the ground of the GTEM, the field level and the induced level along the wire are not constant. To prevent this problem, an angle is given to the system, to maintain a constant coupling mechanism between the fields and the harness (figure 1).

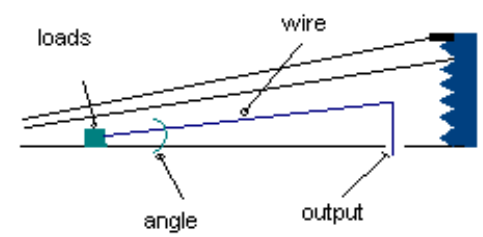

figure 1: GTEM test configuration

\section{Simulation models.}

In automotive standards, the harness is put 5 centimeters upon the ground plane in the HAC (figure 2).

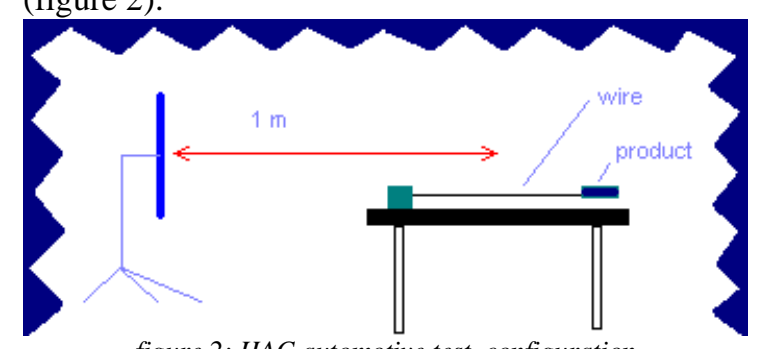

figure 2: HAC automotive test configuration

It constitutes a capacitor per meter. If we consider a single wire, given by:

$$
\mathrm{Zc}:=60 \cdot \ln \left(4 \cdot \frac{\mathrm{h}}{\phi}\right)
$$

Capacitor per meter is:

$$
\mathrm{C}:=\frac{1}{\mathrm{c} \cdot \mathrm{Z}_{\mathrm{c}}}
$$

Starting from this expression, the equivalent current generator which traduces the coupling mechanism between the electric field and the wire, for one Telegraph model cell of $d x$ length is:

$$
\mathrm{i}:=\mathrm{s} \cdot \boldsymbol{\varepsilon} \cdot \mathrm{h} \cdot \mathrm{C} \cdot \mathrm{dx} \cdot \mathrm{E} \cdot \cos \theta
$$

$\mathrm{s}$ is the Laplace operator, $\mathrm{h}$ the height between the wire and the ground plane, $\phi$ the diameter of the 
wire, $\theta$ the angle between the normal vector of the ground plane and $\mathrm{E}$ the incident electric field. The current generator come in parallel with the capacitor. So an equivalent Thevenin model use a voltage generator $\mathrm{hE}=\mathrm{g}$ in serial with the capacitor. The magnetic field coupling model uses the electromotive force (emf) given by the Lentz law, with the impedance of the local inductance of the line is:

$$
\mathrm{e}:=-\mathrm{dx} \cdot \mathrm{h} \cdot \mathrm{s} \cdot \mu \cdot \mathrm{H} \quad \mathrm{L} \cdot \mathrm{dx}:=\frac{\mathrm{Z}_{\mathrm{c}} \cdot \mathrm{dx}}{\mathrm{c}}
$$

Those generators are added to the Telegraphs model of the line. It's only necessary to put the good load at each termination to complete the model. The results obtained with this first approach are quite good but, for the GTEM some modification have to be completed. The functions $\mathrm{h}, \mathrm{L}, \mathrm{C}$ become functions of the length (x). To modelize the cross talk effects between two wires, it is used a classical Telegraph model of cross talking as describe by Vabre[1], with the elements $\mathrm{M}$ ( mutual inductance) and $\gamma$ (mutual capacitance). As we show it after, under the configuration choice, the energy levels estimated with this model was sufficient to predict the disturbance of a equipment, considering that the device susceptibility is known. For example, if we consider an IC input. Knowing the susceptibility threshold in Volt per Volt or Volt per Watt, which is the transmitted power to the differential voltage of the input, we can determine if there is a EMC immunity risk for this input[2] or not. The equivalent impedance of the IC can be measured with a network analyzer, when looking for the S11 parameter. More higher is the frequency, more lower is the influence of the peak incident voltage value to the impedance value. This is often verified for frequencies in the $10 \mathrm{Mhz}-$ $500 \mathrm{Mhz}$ band, which is of our first interest. The layout of the PCB is well known, which modelization is easy. TLine under PSPICE is sufficient to modelize the PCB. So, such a complete simulation including the harness limited to two wires, the generators traducing the coupling mechanism with the fields, and the impedance in common and differential modes, allow to evaluate the levels presented to the IC. Correlation between experiments and simulation are in a 2-3 difference factor which is sufficient to make EMC susceptibility predictions.

\section{Experiments.}

As the height of the septum in the GTEM increase from the beginning to the end of the antenna, even if we consider a homogeneous electric field on the whole length (which is not correct [3]), the induced generators along the harness are not constant. To avoid this phenomenon, an angle is given to the harness. The function to height is $\mathrm{hw}(\mathrm{x})=\mathrm{a} \cdot \mathrm{x}$. To this function correspond an increasing characteristic impedance of the wires. But the electric coupling factor is constant, like the septum follow a function similar of the form $\mathrm{h}(\mathrm{x})=\mathrm{b} . \mathrm{x}$, knowing that $\mathrm{g}=$ hw.E:

$$
\mathrm{g}:=\mathrm{a} \cdot \mathrm{x} \cdot \frac{\mathrm{V}}{\mathrm{b} \cdot \mathrm{x}}
$$

Same approach is obtained for the magnetic field coupling, with the flux surface beginning constant on $\mathrm{x}$, considering the TEM mode for the propagation of the field in the GTEM:

$$
\mathrm{e}:=-\mathrm{dx} \cdot \mathrm{a} \cdot \mathrm{x} \cdot \mathrm{s} \cdot \mu \cdot \frac{\mathrm{V}}{\mathrm{b} \cdot \mathrm{x} \cdot \mu \cdot \mathrm{c}}
$$

The ratio $a / b$ shows a correlation between the field strength in the GTEM compare to the one in the HAC. If we consider a near TEM mode, the higher value of (a) determines this condition. The maximum ratio is around $1 / 20$, with a harness beginning at 5 centimeters of the ground plane. Another source of dispersion is the non homogeneous repartition of the field in the GTEM. Studies were previously made on those aspects[4], and for equipment of our interest (around $200 \times 200$ $\mathrm{mm}$ ) the deviation between the field in the GTEM and the field in a HAC is equal to $6 \mathrm{~dB}$. With such various criteria, the total voltage measured on the loads, for various values of impedance's, can be compared with the same results in HAC. Figure 3 and 4 show the simulation and measures for one case of impedance in GTEM and HAC.

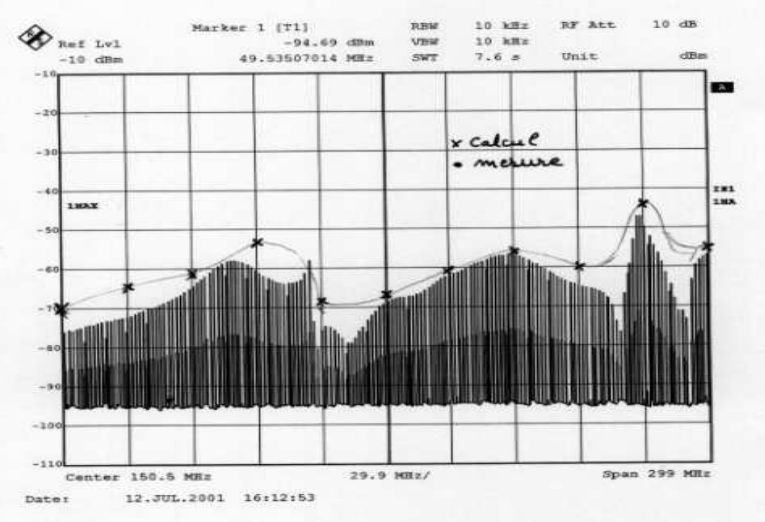

Figure 3: comparison measures/calculus in GTEM

Conclusion for immunity.

To guarantee the correlation, has to be considered the worst case of field strength. We are convinced today that the correlation is true for the immunity of such an equipment in GTEM and HAC. Moreover, during observation of an equipment behavior in GTEM, first, both electric and magnetic coupling are present, and second it's sometimes more representative from the real coupling mechanism with the field propagating along the harness. To reach this, an angle is given to the harness with the ground of the GTEM, with a 5 centimeters distance at the beginning and a 10 centimeters distance minimum at the end. The bigger equipment is 
placed where the septum is higher, on the "front wave" side of the coupling.

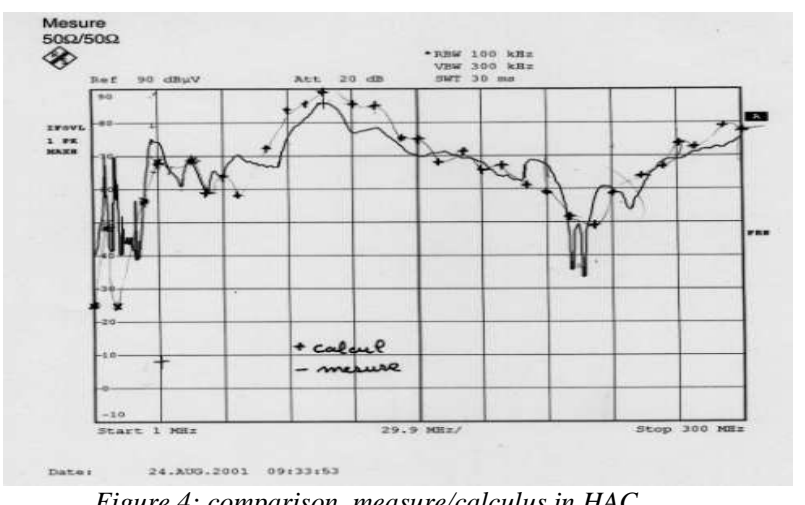

The missing information to make good prediction for an equipment EMC compliance is the detection efficiency of the IC, and its wide band susceptibility. Know how on back version often gives first order information about the power level not to reach in differential mode to avoid to be disturbed. Considering two wires only is not a critical point, as we look for the worst cases where the coupled energy can not be distributed to many wires.

\section{Emissions aspects.}

Concerning the frequency band, and the load condition of a line, the models to use to calculate the coupling between a wire and the GTEM can be quite different. They respect all the same general formula, but some terms can be neglected depending of the frequency range. In a first step we use a matched line, with the same length as the one used in the standards, but with a matched stage to avoid standing waves. The extremity is alternatively in short circuit or open circuit. The wire is placed in the center of the GTEM, then the S21 ratio parameter is measured, connecting the input of the GTEM to the input of a network analyzer. Then we can compare the curve to the simulation. This transfer function can be used after, knowing the noise generator, as ICEM model for an IC to evaluate the emission level of an equipment in the GTEM and in HAC. A first difficulty is to characterize the emission at low frequency, where far field model are not more usable. A difference with the immunity approach when looking for the order of level we reach in standard is that, in some cases, the direct emissions coming from the IC's are not negligible. Both harness and IC radiated emissions must be considered. We look first for the harness emissions, and after we discuss of the direct IC emission. One more time we can wonder if the same facility could not be used for both radiated immunity and emission, even if there is diversion. In a development phases, the results can give good order of level to evaluate the compliance of an equipment or a device.
Wire emission threw S21 parameter.

If the load is an open circuit, below $50 \mathrm{MHz}$ for a 1,5 meter long line, the equivalent model for the source constituted by the wire is a capacitor, which value is determine by the same way as for immunity model (formula 2). For open circuits, the calculus can be limited to the electric current in this capacitor, the conducted current can be not considered. From this current we can evaluate the vector potential in a Coulomb gauge, projected on the TEM mode of the GTEM, and after the electric field:

$$
\vec{a}=\frac{-\mu \cdot I \cdot e^{j w t} \cdot e^{-j \beta r} \cos (\theta)}{4 \pi r} \quad|\vec{E}|=\left|\frac{-\partial a}{\partial t}\right|=j w a
$$

Having the electric field in all the space in the GTEM, we can integrate it on the high for each (x) element of the length. The equivalent electric schematic of the coupling mechanism of the field with the TEM mode of the GTEM is:

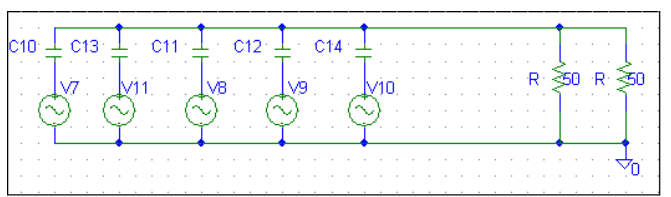

Each voltage generator is linked to the local electric field work with the distance between the ground and the septum. The voltage measured at the input of the GTEM is:

$$
V=\frac{\sum_{x} V(x) j C(x) w}{\sum_{x} j C(x) w+\frac{1}{50}+\frac{1}{50}}
$$

$\mathrm{V}(\mathrm{x})$ is determined by the integration of the derived part of the vector potential, modified by a special function to take into account the limit condition due to the GTEM walls (comparison measure/calculus figure 5).

$$
V(x):=\int s \cdot \vec{s} \cdot \overrightarrow{a(x, y, z)} d y \cdot\left[1-e^{\left(-s \cdot \frac{m}{c \cdot 2}\right)}\right]
$$

In short circuit extremity condition, the magnetic field emission is first observed, where for this case the wire is compared to an inductance. Knowing the conducted current on the wire, with the hypothesis that the electric current is negligible it's possible to evaluate the magnetic field emitted by the wire using the Biot \& Savart formula, in all the space included in the GTEM, and by taking account of the magnetic field rotation in azimuth. So, it's possible 
to calculate the electromotive force induced by length dx:

$$
e(x):=\int_{0}^{h(x)} \int_{0}^{L} \frac{\vec{\mu} \cdot \overrightarrow{d i} \cdot \overrightarrow{d l} \times r^{1} \cdot z}{4 \cdot \pi \cdot r^{2} \cdot \sqrt{x^{2}+z^{2}}} d z d y
$$

$\mathrm{L}$ is the width of the GTEM, $r$ the distance between the current element di of length $\mathrm{dl}$ and the point in the volume where the field is calculated. Integration of $\mathrm{e}(\mathrm{x})$ on $(\mathrm{x})$ gives the value of the local emf. So, an equivalent schematic for this kind of coupling is:

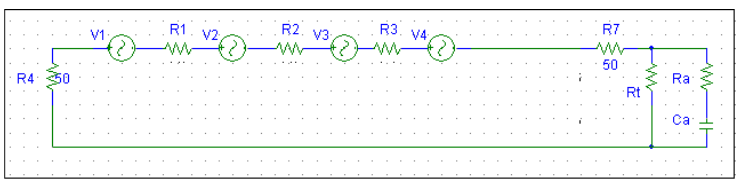

The comparison between measure and calculus with those models gives a good correlation, as shown figure 6 .

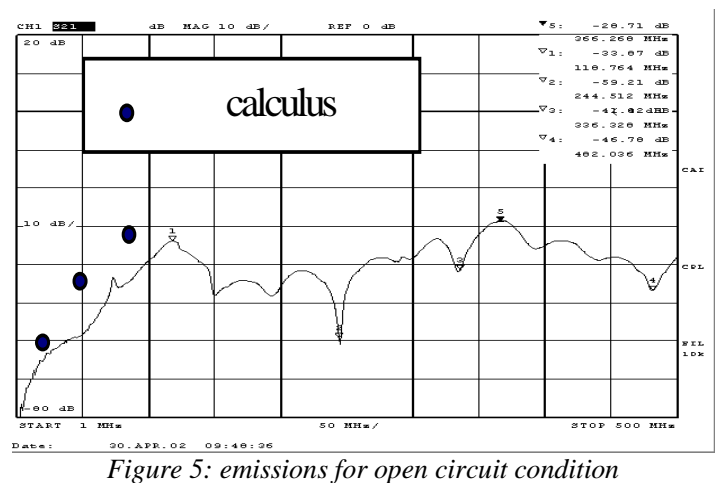

Another part of emission comes from the direct electrostatic field emitted by the wire to the septum, or the quarter wave antenna in case of the standard test. The charge acting as sources for this field are given by $\mathrm{C}$. V considering the functional potential $\mathrm{V}$ constant along the wire, for the frequency of interest, coming from the Fourier transformation for a the functional temporal signal of the equipment, $\mathrm{C}$ is the lineïc capacitor of the wire upon the ground plane.

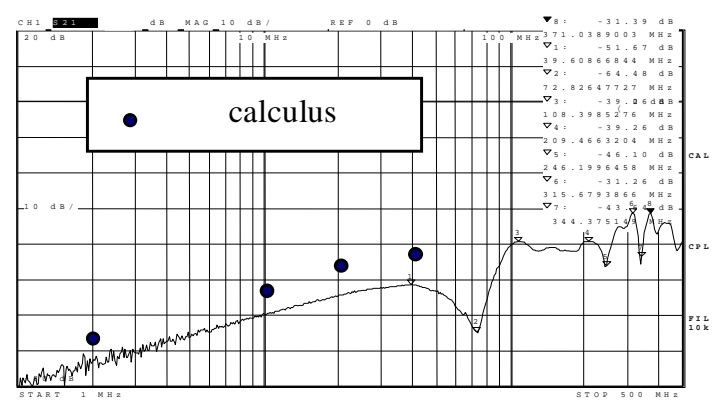

Figure 6: emissions for short circuit condition
The field value at a point of the antenna is given by:

$$
E=-\frac{C V}{\varepsilon}\left[\int_{-L / 2}^{L / 2} \frac{\partial}{\partial y}\left(\frac{1}{4 \pi \sqrt{x^{2}+z^{2}}}\right) d z\right]
$$

For a wire of length $\mathrm{L}$, in the (z) direction of space. (x) is the space distance between the wire and the antenna, and (y) the direction of the antenna axes. This kind of radiation can be the major one for frequencies below $1 \mathrm{MHz}$ in standard measure. For the GTEM it gives the part of the voltage coming from the direct coupling capacitance between the wire and the septum. But it's less than the low frequency magnetic field part of radiation, which not appear in standard tests.

Knowing the S21 parameter for the GTEM, it is possible to predict the equipment emissions with the devices noises source. An ICEM model for noise is known. Calculating the Fourier transformation of this temporal signal, to use the same formulas as the ones used to obtain the S21 response, it's possible to calculate the emission of the equipment in the GTEM. The wiring part for the radiation, and the actions to do to reach the compliance for an equipment are elements that can be determined in GTEM, in relatives. The real emission performance is known only after a measure in HAC, because it stills to must difference in the two facilities to be able to guarantee, not in a worst case, the radiation's levels seen in HAC, knowing the ones seen in GTEM.

Regarding the direct device emissions, some works have already been done [5]. This previous work shows two very interesting conclusions: firstly, the correlation between near fields scan and GTEM gives less than $5 \mathrm{~dB}$ dispersion. Secondly, the comparison with measure in free space show a $75 \%$ correlation, with dispersion less than $10 \mathrm{~dB}$. Actually, making a link between the IC and a group of electric and magnetic moments, can be evaluated. First, we consider the electric and magnetic fields in free space conditions (see annex). Concerning the electric field we have:

$$
\overrightarrow{|E|}=\frac{\mu \omega^{2}}{4 \pi r} \operatorname{Cos} \Theta \cdot P_{0 z}
$$

Poz is the electric moment of the equivalent dipole, defined by the product Q.1. Concerning the magnetic field evaluated for a rectangular loop:

$$
\left|\overrightarrow{H_{y}}\right|=\frac{M y \cdot \omega}{\pi^{2} r^{2} c}
$$

My is the magnetic moment of the equivalent dipole, ixS. Making a comparison between those expressions and the ones obtain for the field in a 
GTEM, once can deduce equivalent dipoles, for each polarization of a device, starting from GTEM or TEM measures (this was well demonstrate in [6]). But, if this approach is sufficient for a far field evaluation, it doesn't give any information for a near field coupling mechanism between the device and another PCB or device on the same PCB. To have a sufficiently good image of the field, some recents studies, more particularly in near field scan shows that the dipoles models are not usable, because a global dipole model doesn't give information of the current distribution in the chip, and on a more detailled radiation diagram. So, what can give the GTEM or TEM characterization, and how to increase an ICEM model to predict near field coupling? We have shown that a good knowledge of the relation between the field emitted and the power measured by TEM or GTEM facility allow to use completely those facilities to predict and estimate EMC compliance in the one meter HAC test. This evaluation can be too simply extractions from relatives results of emission between differents IC's or some IC's and a reference one. To give a definition of this aspect for one device, the dipole model can be a good way. Defining a space reference attached to the package, the characterization could be a group of six values of equivalent dipoles. To go deeper in the source description of the emitted field, we can consider the currents identified in the ICEM model. By application of those currents on the harness wires, we can estimate the field radiated by the harness. After a comparison with the measure, once can estimate if the direct field emitted by the device is of first influence or not (by direct radiation we must understand the radiation coming from the bonding and package lead elements). In the estimation, both electric and magnetic fields must be calculated. In another way, if we don't have the ICEM model, once can just compare the field received with the device alone (on its test PCB with reduced tracks emissions), or by the harness connected to the device. Now, why couldn't we be sure of the correlation with the results we will obtain in HAC facility after such a study in GTEM or TEM? Hypothesis of others currents repartition can explain some difference [7]. But a very detailled calculus of all the coupling mechanism in GTEM or TEM, including capacitive coupling between current device's loop and the septum should gives energy detectable on the measure side. Another reason concern the tracks direction on the PCB. Depending on their direction compare to the HAC antenna one, the results can be very different between GTEM, TEM and HAC, and we know that tracks and bonding elements are of first influence in this radiation [8]. According to the harness configuration (distance signal wire, ground wire), relatives influence of harness and chip on the total radiation seen is modulated. In case where all the elements are perfectly describe, the correlation between GTEM and HAC is possible, and that's what we have done in fact in some simple cases.

Conclusion about emissions aspects.

The GTEM cell can be used to confirm behavior or to search for improvement of the equipment performance. The difficulty comes from the special actual situation in automotive where levels targeted are so low that it's impossible with the actual technologies and cost wished, to have a sufficient margin. Thus, the uncertainty between the results in GTEM and in HAC, coming from systems definition unaccuracy, are too big to have a good control of the compliance of the project. Anyway GTEM is still a very good facility to increase the compliance for an equipment working in a relative way. That's often the working way used for TEM facility when using results of measurements made by IC manufacturers. Waiting for more information contains perhaps tomorrow in a global ICEM model, automotive suppliers have this only way to predict EMC emission risk from IC's.

\section{References.}

[1]: J.P.Vabre. «Electronique des impulsions » Ed.Dunod.

[2] : O.Maurice. «Susceptibility of digital device to high frequency pulses ». Radec's 1997.

[3]: D.Hansen \& all. "Sources of problems in the GTEM structure and recommended solutions". Euro EMC 1996.

[4]: S.Ishigami \& all. "Estimation of E-field distribution in a loaded GTEM cell".

[5]: K.P.Slattery. "A comparison of the near field and far field emissions of a pentium clock IC".

[6]: R.De Smedt \& all. "Correlation between direct radiation and TEM cell measurement of an active test chip". EMC Europe 2000.

[7]: F.Fiori \& all. "Weakness of the TEM cell method in evaluating IC radiated emissions".

[8]: R.De Smedt \& all. " TEM cell measurement of an active EMC test chip". IEEE EMC 2000 Symp.

$$
\begin{aligned}
& q=q_{0} e^{j w t} \quad \vec{a}=\frac{-\mu j w q}{4 \pi r} e^{-j \beta r} \cos \Theta \\
& \vec{E}=\frac{-\partial \vec{a}}{\partial t} \\
& \overrightarrow{a_{z}}=\frac{\mu i 2 a}{4 \pi} e^{-j \frac{w r}{c}}\left\{\frac{e^{j 2 \pi \frac{a}{\lambda}}}{r-a}-\frac{e^{-j 2 \pi \frac{a}{\lambda}}}{r+a}\right\} \cdot \vec{z} \\
& H_{y}=\left\{-2 \frac{M_{y}}{\pi^{2} r^{3}}-j \frac{w}{c} \frac{M_{y}}{\pi^{2} r^{2}}\right\} \cdot e^{-j \frac{w r}{c}}
\end{aligned}
$$

